\title{
Giant left atrium in undersized mitral ring annuloplasty
}

\author{
Nicole Bertin', Aniello Pappalardo², Alessandro Minati², Gabriella Forti², Serena Favretto³, \\ Franco Pertoldi4, Eleonora Ficarra4, Francesco Pelizzo4, Olga Vriz ${ }^{4}$ \\ 1 Internal Medicine, Department of Experimental and Clinical Medical Sciences, University of Udine \\ 2 Department of Cardiac Surgery, University of Trieste \\ 3 Department of Translational Medicine, University of Eastern Piedmont, Novara \\ 4 Department of Cardiology and Emergency Medicine, San Antonio Hospital, San Daniele del Friuli (UD), Italy
}

\begin{abstract}
Enlargement of left atrium occurs in patients with longstanding mitral valve disease due to chronic pressure and volume overload and occasionally left atrium reaches a massive enlargement, condition known as giant left atrium. It is most commonly associated with rheumatic mitral valve disease, both stenosis and regurgitation. This unique case deals with a 70-year-old woman who developed a giant left atrium due to a severe mitral regurgitation from complete prolapse of both mitral leaflets, as a consequence of previous undersized mitral ring annuloplasty.
\end{abstract}

\section{Introduction}

Giant left atrium (GLA) is a rare condition usually associated with longstanding rheumatic mitral disease and rarely observed in patients with other valve disease, because both pressure and volume overload of

Corresponding author: Nicole Bertin, Internal Medicine, Department of Experimental and Clinical Medical Sciences, University Hospital of Udine, Piazzale Santa Maria della Misericordia 15, 33100 Udine, Italy.

Tel. +39.349.1860166. E-mail: bertin.nicole@libero.it

Key words: Giant left atrium; mitral valve regurgitation; mitral valve replacement.

Acknowledgments: We sincerely thank all clinical personal at the participating hospital for their performance and patient care.

Authors' contribution: AM, GF, manuscript writing, intra-operative imagines collecting; FP, radiological imagines collecting; NB, SF, EF, OV, manuscript writing; AP, FP, study supervision. All authors read and approved the final manuscript.

Conflict of interest: the authors declare no conflict of interest.

Received for publication: 08 August 2016

Accepted for publication: 29 August 2016

(c) Copyright N. Bertin et al., 2016

Tipografia PI-ME Editrice, Italy

Monaldi Archives for Chest Disease Cardiac Series 2016; 86:763

doi: 10.4081/monaldi.2016.763

This article is distributed under the terms of the Creative Commons Attribution Noncommercial License (by-nc 4.0) which permits any noncommercial use, distribution, and reproduction in any medium, provided the original author(s) and source are credited. the left atrium and weakening of its wall due to rheumatic pancarditis are involved in its development [1]. Here we present a case of GLA in a patient with previous undersized mitral ring annuloplasty.

\section{Case Report}

A 70-year-old woman presented with a 2-month history of progressively worsening shortness of breath and bilateral peripheral edema. The patient had a known history of atrial fibrillation and previous mitral and tricuspid annuloplasty in 2001 (34-mm and 32-mm Carpentier-Edwards, respectively) for severe non rheumatic mitral regurgitation. She underwent regular follow-up until 5 years ago with echocardiographic evidence of moderate residual mitral regurgitation and severe left atrium dilatation (volume $200 \mathrm{ml}$ ) and then she had no more cardiac check-up. At the present evaluation on physical examination she had elevated jugular venous pressure, holosystolic $4 / 6$ murmur at the apex, bibasilar crackles of the lungs and 3+ peripheral edema. Transthoracic echocardiography (Figure 1) showed a GLA (volume $1262 \mathrm{ml}$, diameter $16.6 \times 12.0 \times 15.0 \mathrm{~cm}$ ) with a severe mitral regurgitation due to complete prolapse of both mitral leaflets. Tricuspid valve was severely incompetent with an estimated pulmonary systolic arterial pressure of $85 \mathrm{mmHg}$. Pulsed wave Doppler at the level of pulmonary valve showed a reduced acceleration time $(78 \mathrm{~ms})$ with a mid-systolic notch in the ascending portion of the signal. Thoracic computed tomography (Figure 2) confirmed the GLA, which occupied mostly of the anterior left thoracic cavity and partly of the right one. It also demonstrated pleural effusion and widening of the carina. Surgery was performed using cardiopulmonary bypass by femoral vessels cannulation. The re-sternotomy was executed with extracorporeal circulation to prevent injury of cardiac structures due to the strict adhesions from previous cardiac surgery. Extracorporeal circulation was then stopped and lysis of scar tissue, which was very difficult because of cardiomegaly and the anatomic displacement of cardiac structures due to the GLA (Figure 3), was performed. At the end of the lysis, the extracorporeal circulation was restarted and conducted with moderate hypothermia with both anterograde and retrograde blood cardioplegia. To enter in the left atrium a trans septal approach was performed. At surgical exposure mitral valve was totally prolapsed and then replaced with a bioprosthesis (Edwards Magna 31 ); the partial detachment of the septal part of tricuspid prosthetic ring could otherwise be repaired without valve replacement. Discontinuation of cardiopulmonary bypass occurred without any problem, with aortic cross-clamping and extra corporeal circulation times of 92 and 228 minutes respectively. The patient got over surgical procedure without complications.

Follow-up by transthoracic echocardiography two weeks after surgery showed a well-functioning prosthetic valve and normal pulmonary 
a

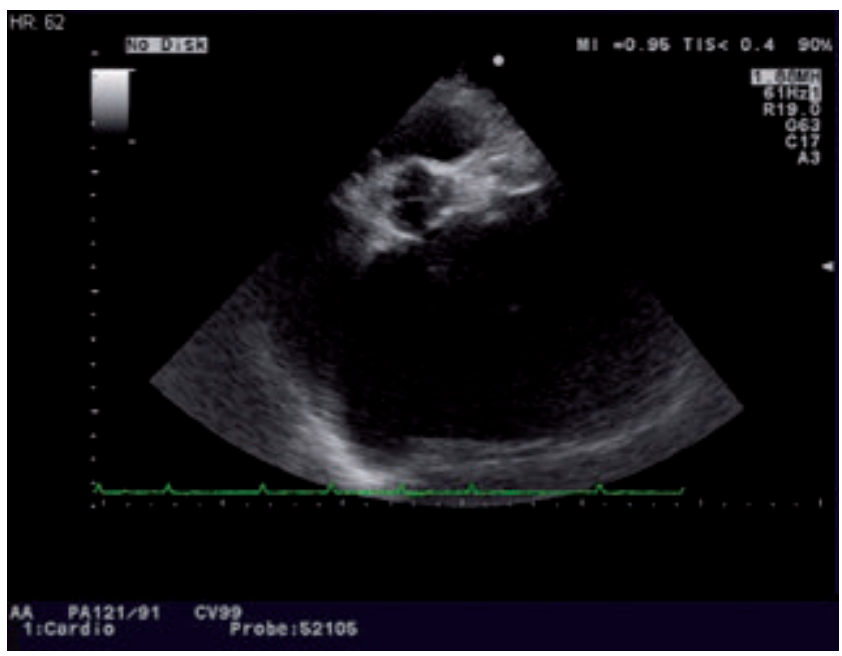

c

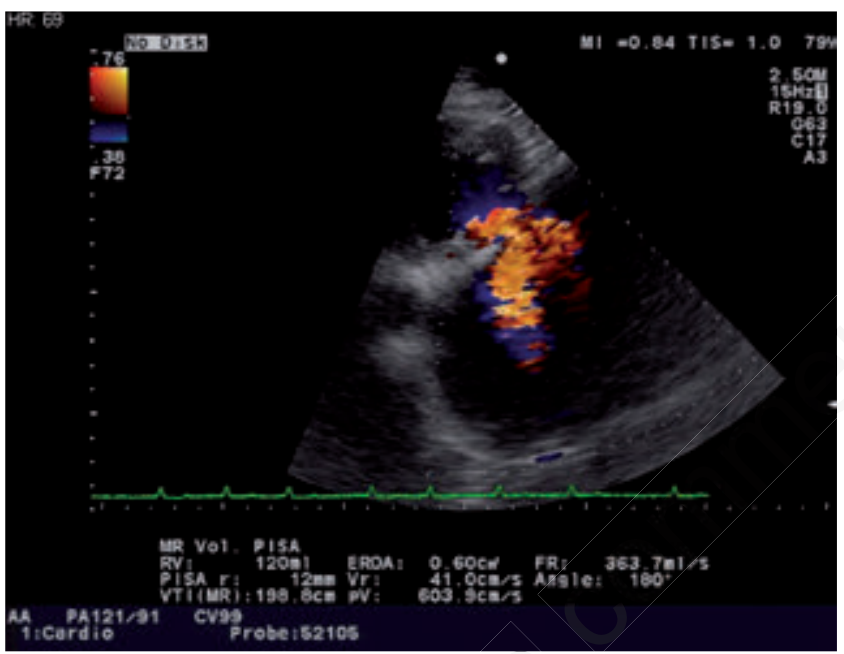

b

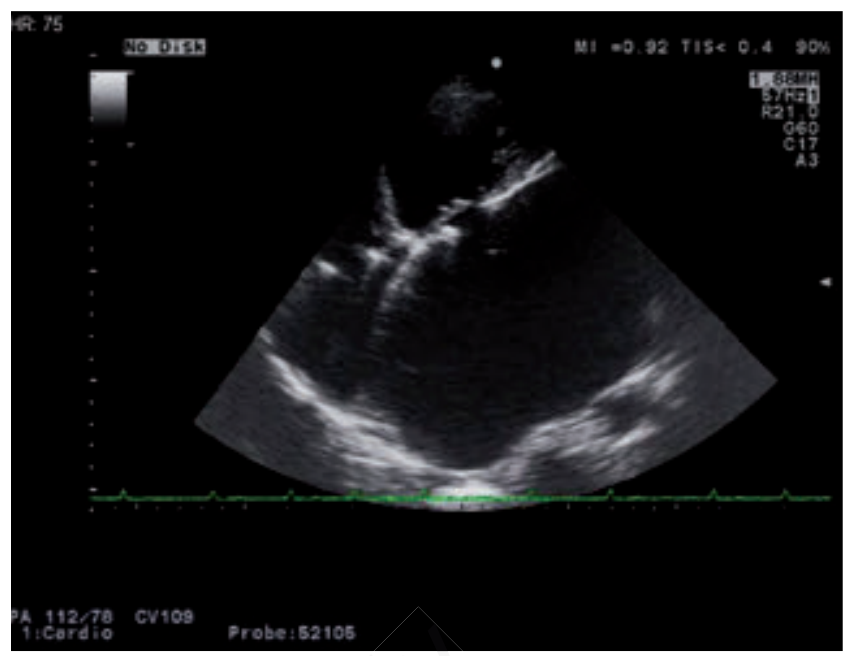

d

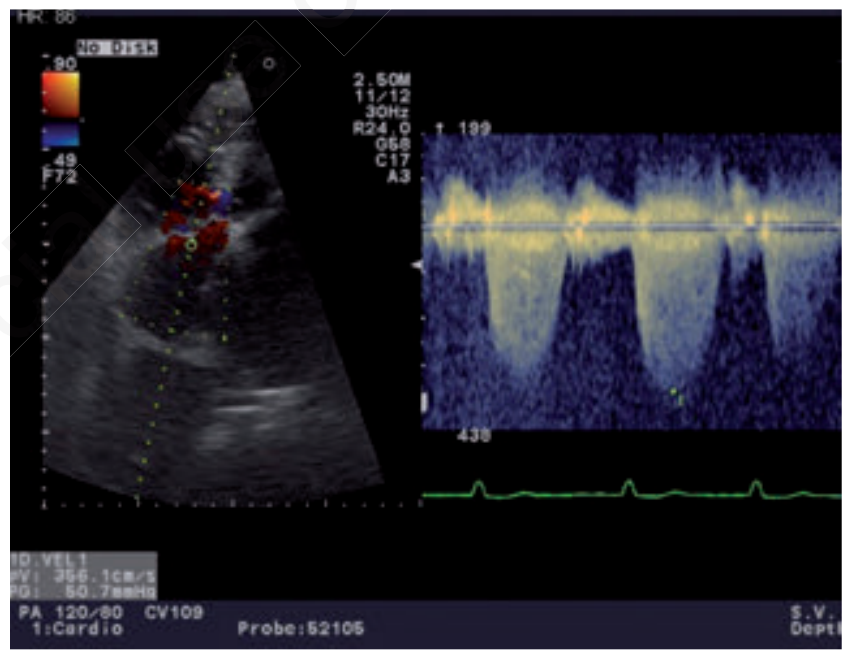

Figure 1. Transthoracic echocardiogram view with: (a) giant left atrium from parasternal short axis; (b) giant left atrium from apical four chamber; (c) severe mitral regurgitation; (d) severe tricuspid regurgitation.

systolic arterial pressure; left atrial was still hugely enlarged. The latest echocardiography control, four months after surgery and with appropriate diuretic therapy, showed a left atrium enlarged (volume $627 \mathrm{ml}$ ) but reduced compared to the pre-surgery dimension.

\section{Discussion}

Various definition of GLA are reported. According to Hurst et al., GLA is defined as one that touches the right thoracic wall at chest X-ray and the condition is frequently caused by rheumatic mitral valve disease [1]. Isomura and coworkers define GLA as left atrium larger than $6 \mathrm{~cm}$ in diameter [2]. Piccoli et al. consider GLA as a cardio-thoracic ratio on chest X-ray of $>0.7$ in addition to a left atrial anterior-posterior diameter of $>8 \mathrm{~cm}$ on transthoracic echocardiography [3].
Massive enlargement of left atrium has a reported incidence of $0.3 \%$ and it is usually associated with longstanding rheumatic mitral disease, both stenosis and regurgitation, but its etiology is complex and not fully understood. Chronic mitral valve disease leads to increased intracavitary pressure and volume overload of left atrium with its subsequent enlargement. Otherwise, GLA is rarely observed in patients with non-rheumatic mitral valve involvement, because rheumatic pancarditis damages the entire heart by causing chronic inflammation and weakening its tissues, including the left atrial wall that then dilates more easily [1]. GLA can be associated with various complications, including atrial fibrillation, increased pulmonary venous pressure, symptoms related to compression of the esophagus and airways, Ortner's syndrome, thrombus formation, thromboembolic events and sudden death. In rare cases, patients may remain asymptomatic. 
a

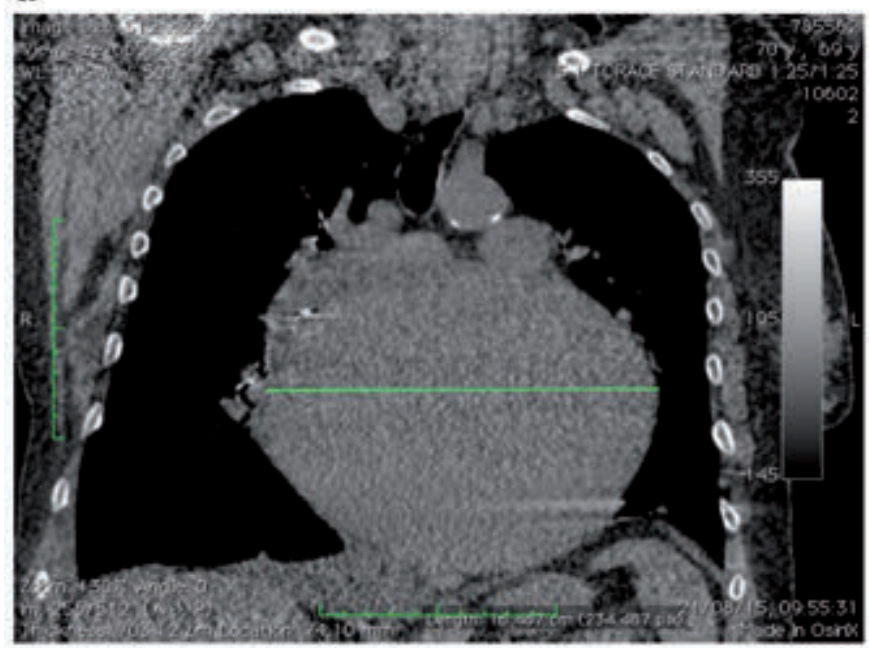

c

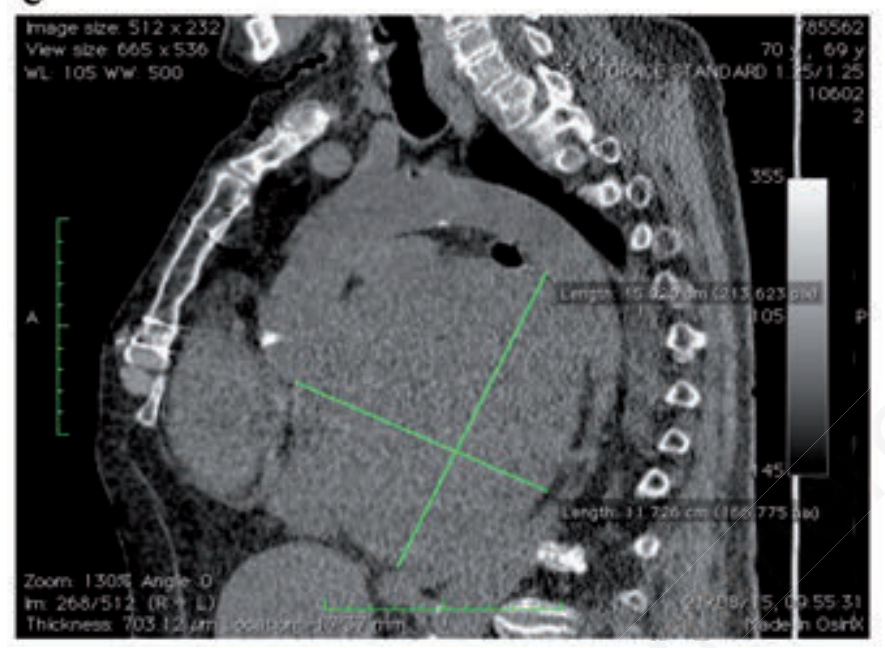

b

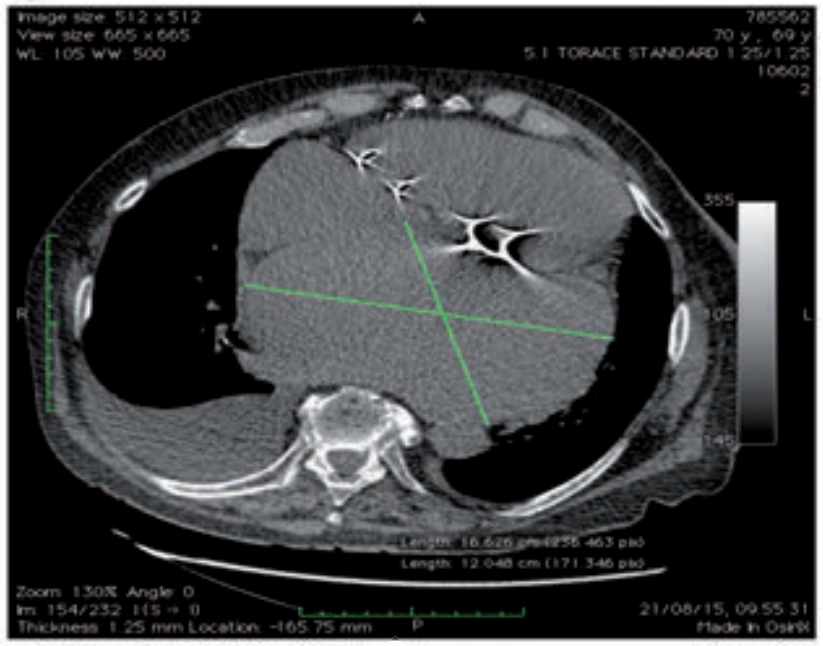

d

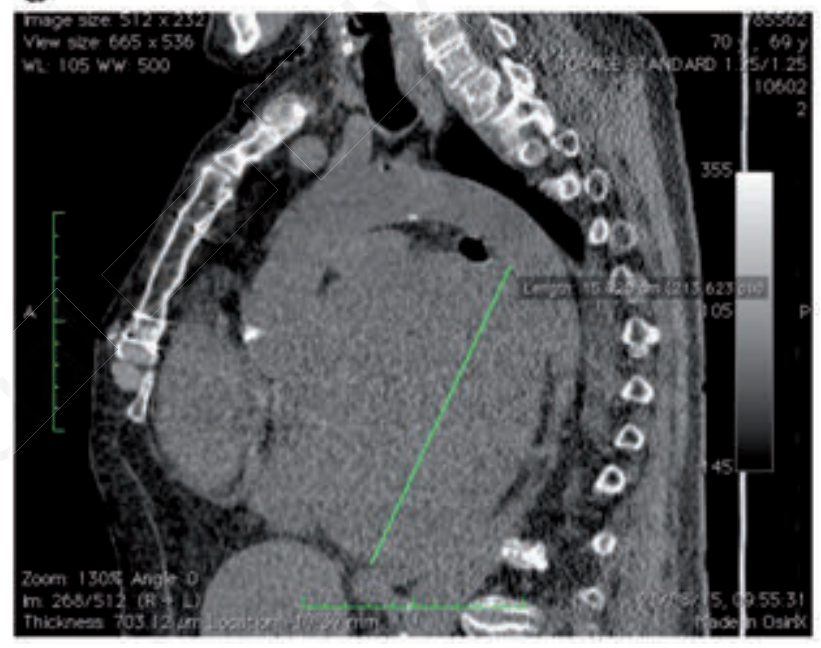

Figure 2. Preoperative thoracic computed tomography showing giant left atrium occupying mostly of the anterior left thoracic cavity and partly of the right one, from: (a) coronal reconstruction; (b) axial reconstruction; (c) and (d) sagittal reconstructions.

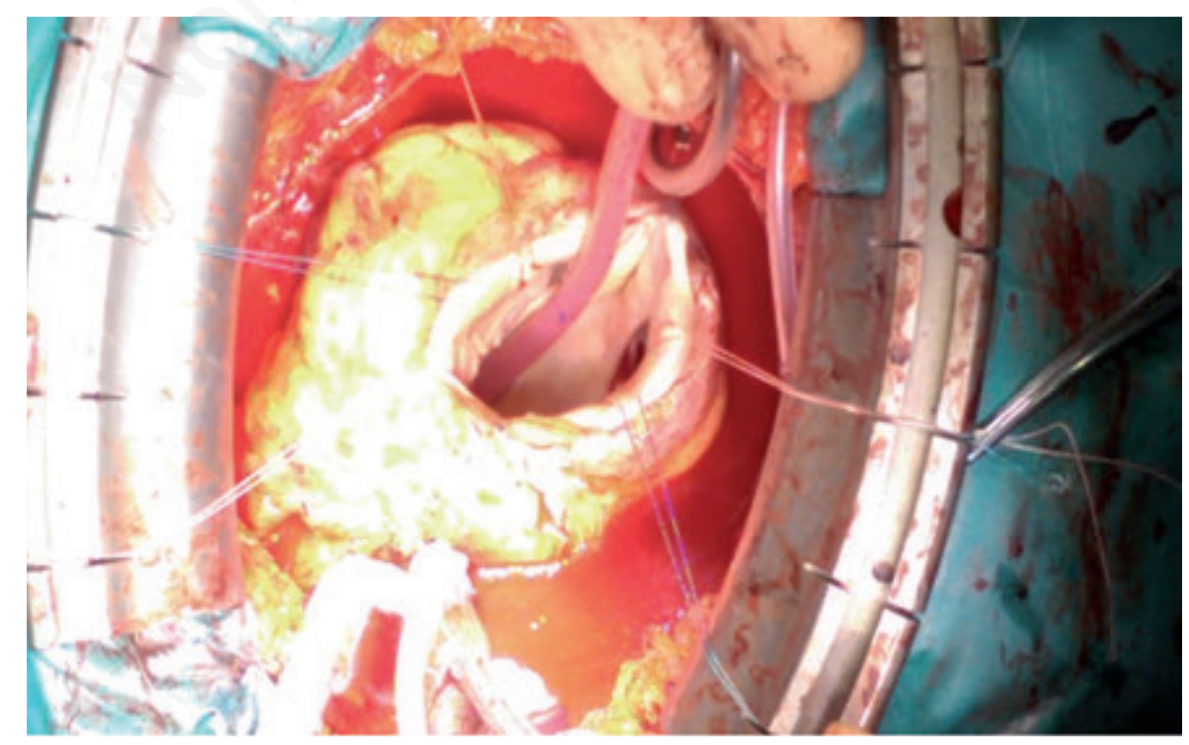

Figure 3. Giant left atrium by trans septal approach: intra-operative view. 
To the best of our knowledge, the largest ever GLA reported $19.3 \times 14.7 \mathrm{~cm}$ was due to severe rheumatic mitral regurgitation [4]. In our case mechanism underlying progressively worsening of mitral regurgitation and left atrial enlargement was an undersized mitral ring annuloplasty leading to complete mitral valve prolapse and severe mitral regurgitation.

Surgical management of GLA usually takes place during mitral valve surgery. The simplest technique for left atrium volume reduction is partial plication (or resection) of inferior atrial wall; other current techniques are partial plication or resection of both inferior and superior atrial walls and partial heart auto-transplantation [5]. However there are still controversies regarding surgical management of GLA and effectiveness of surgical options [6,7].

Surgical mortality in patients with mitral valve disease and GLA is high and ranges from 7 to 20\% [3], and other post-operative complications include low cardiac output syndrome and respiratory insufficiency. Moreover many surgeons claim that successful mitral valve surgery alone will result in left atrial remodeling and size reduction and so they do not often perform left atrial volume reduction during valve surgery.

In our case the additional factor to consider was that the patient underwent to a second mitral valve surgery. This procedure has a higher mortality compared to first-time operation, in particular factors such as major cardiovascular iatrogenic injury at re-entry and incremental cardiopulmonary bypass time are associated with higher operative mortality [8]. To avoid these complications, especially the high risk of cardiovascular iatrogenic lesions at re-entry due to the anatomic displacement of cardiac structures and tenacious adhesions from prior cardiac surgery, surgical reduction of GLA was not performed. The left atrium volume reduction, after 4 months surgery, was not due to remodeling (probably not feasible because of the longstanding of mitral regurgitation) but related to important congestive heart failure therapy.

\section{Conclusions}

Undersized mitral ring annuloplasty was not only irrelevant but was responsible of the worsening of mitral regurgitation which led to huge left atrium and then severe congestive heart failure. Although a second surgical re-correction was bearing high risk, it was mandatory to replace the mitral valve and to repair the tricuspide valve.

\section{References}

1. Hurst JW. Memories of patients with a giant left atrium. Circulation 2001;104:2630-2631.

2. Isomura T, Hisatomi K, Hirano A, et al. Left atrial plication and mitral valve replacement for giant left atrium accompanying mitral lesion. J Card Surg 1993;8:365-370.

3. Piccoli GP, Massini C, Di Eusanio G, et al. Giant left atrium and mitral valve disease: early and late results of surgical treatment in 40 cases. J Cardiovasc Surg (Torino) 1984;25:328-336.

4. Ozkan A, Tuncer A, Ozkan M. Giant left atrium. J Am Coll Cardiol 2012;60:e13.

5. Apostolakis E and Shuhaiber JH. The surgical management of giant left atrium. Eur J Cardiothorac Surg 2008;33:182-190.

6. Sinatra R, Pulitani I, Antonazza A, Melina G. A novel technique for giant left atrium reduction. Eur J Cardiothorac Surg 2001;20:412-414.

7. Lessana A, Scorsin M, Scheuble C, et al. Effective reduction of a giant left atrium by partial autotransplantation. Ann Thorac Surg 1999;67:1164-1165.

8. Onorati F, Perrotti A, Reichart D, et al. Surgical factors and complications affecting hospital outcome in redo mitral surgery: insights from a multicenter experience. Eur J Cardiothorac Surg 2016;49:e127-133. 